\title{
HUMILLADOS Y OFENDIDOS: UN ESTUDIO ACERCA DEL DESPRECIO Y LA DISCRIMINACIÓN EN EL PERÚ ${ }^{1}$
}

\author{
Ramón León ${ }^{2}$ \\ Universidad Ricardo Palma y Universidad de Lima \\ Juan José Tan Martínez \\ Universidad Ricardo Palma
}

Setecientos ochenta y nueve universitarios peruanos 362 varones : 427 mujeres, en edades entre 16 y 27 años) respondieron una escala de 6 items que evaluaba la percepcion de desprecio en el Perú. Aunque no se die: is diferencias estadísticamente significativas entre los puntajes totales de hombres y mujeres, el análisis de items puso en evidencia esas diferencias cn tres de ellos. Los sujetos que se vieron a si mismos como de raza blanca tuvicron una percepción más fuerte de desprecio en la sociedad peruana que otras personas. la validacion y otras cualidades psicomćtricas de la Escala de Percepción de Desprecio en e) Perú son comentadas en esta comunicación.

Palabras claves: Percepción de desprecio en el Perí.

Humiliated and offended people: A study about scorn and discrimination in Peru.

Seven hundred eighty nine Peruvian students $(362$ men and 427 women, in ages between 16 and 27 years) answered a 6 -items Scale of Perception of Contempt in Peru. Although there is no significative statistical differences between the total scores in men and women, item- analysis showed these differences in three items. The subjects wich saw themselves as of white race had a stronger perception of contempt in the Peruvian society than the other people. The validation and other psychomethric qualitics of the Scale of Perception of Contempt in Peru are commented in this communication.

Key words: Perception of Contempt in Peru

1 Los autores agradecen a la Fundación Konrad Adenauer (Konrad - Adenaner - Stiftund, de Bonn, República Federal de Alemania, por el apoyo prestado para la realización de cste trabajo, así como al Sr. Jorge Bazán, quien llevó a cabo el tratamiento estadístico de los datos; y a la Srta. Kathia Murillo S., que ayudó en la obtención de la muestra.

2 Profesor Asociado de la Lniversidad Ricardo Palma y de la Iniversidad de lima. Obtuvo su licenciatura en la Universidad Garcilaso de la Vega y el Doctorado en la Universidad do Wurburgo (Acmania). Ha publicado diversas investigaciones en el área de la psicologia social. Dirección L.R.P.: Ar. Benavides cdra. 52 s/n, Lima 33.

3 Licenciado en psicología de la U.R.P., donde actualmente se desempeña como jefe de práctica. 

En los años ochenta el Perú dejó de ser un país "en el que no ocurría nada y en el que todo invitaba al tedio" (Flores Galindo 1988; p. 232) para ganar paulatinamente la atención de círculos periodísticos, académicos y políticos internacionales.

Las causas: de un lado, la presencia cada vez más frecuente y cruenta de Sendero Luminoso y la inflación desbocada en los tramos finales del gobierno de Alan García. A esto se agregó el sorprendente triunfo electoral de Alberto Fujimori; y la implantación, tras su ascensión al poder, de un severo programa económico.

Obviamente, no sólo los extranjeros se interesaron por lo que sucedia en el Perú. Los primeros en hacerlo fueron los propios peruanos especialistas en ciencias sociales, quienes llegaron a la conclusión de que los parámetros de interpretación de la realidad nacional hasta entonces considerados como válidos habían perdido su valor.

Son de esos años algunas obras que ensayan una aproximación más realista al drama del Perú: La crisis del estado patrimonial en el Perí (Stein \& Monge 1988); Violencia estructural en el Perí (Asociación Peruana de Estudios e Investigación para la Paz, 1990); Violencia y pacificación (Comisión Especial del Senado sobre las Causas de la Violencia y Alternativas de Pacificación en el Perú, 1989); En qué momento se jodió el Perí (Milla Batres, 1990); Desborde popular y crisis del estado (Matos Mar, 1987); Buscando un inca (Flores Galindo, 1994), se encuentran entre las más leídas.

Estos y otros trabajos analizaron desde diversas perspectivas nuestra desafiante realidad, tomando en consideración fenómenos psicosociales hasta entonces por lo general ignorados o negligidos: el racismo (Flores Galindo, 1994); la profunda desconfianza interpersonal (Milla Batres, 
1990); la violencia estructural (Asociación Peruana de Estudios e Investigación para la Paz 1990; Comisión Especial del Senado sobre las Causas de la Violencia y Alternativas de Pacificación en el Perú, 1989); el arribismo (Stein y Monge, 1988), etc.

La imagen del Perú surgida del aporte de estos estudiosos fue la de una sociedad escindida por una fractura esencial, que ha impedido la cristalización de lo que Jorge Basadre llamó "la promesa de la vida peruana" (Basadre, 1958).

Crasas diferencias económicas, racismo, autoritarismo, entre otros elementos de una realidad signada por la injusticia, provocan entre los peruanos un desencuentro radical, una verdadera sensación de extrañeza que torna virtualmente imposible la comunión de sus esfuerzos, impidiéndoles verse y sentirse como iguales en dignidad más allá de las mil diferencias que naturalmente existen entre los individuos. La conciencia, pero sobre todo el sentimiento de igualdad (igualdad en dignidad; igualdad en deberes, en derechos, en oportunidades y posibilidades), fundamentales en toda democracia que legítimamente pueda autocalificarse como tal, han sido realidades carenciales en el Perú a lo largo de toda su historia. El sentirse extraños entre sí, de otro lado, impide el desarrollo del sentimiento de solidaridad, central en la constitución de una identidad grupal, pues "la mirada extraña es por sí misma y de todos modos también una mirada limitada emocional y culturalmente" (Heinrichs, 1997; p. 55).

No sorprende, por ello, que en una "república sin ciudadanos" (Flores Galindo, 1994); en un país en el cual hay ciudadanos de primera y segunda categorías (Montoya, 1992), y, como recientemente lo ha señalado López (1997), "ciudadanos reales e imaginarios", surja y se exprese un sentimiento de consecuencias dramáticas para las relaciones interpersonales: el desprecio.

Es verdad que en los últimos veinte años muchas cosas han cambiado en la sociedad peruana y que hoy se observa "una tendencia general a 48 
que la gente sea más 'igualada', más horizontal en el mejor sentido de la palabra" (Ames, 1995; p. 49). Sin embargo, el trato desigual, casi perpendicular, entre los peruanos, sigue siendo un fenómeno de alarmante frecuencia.

"Bajo diferentes formas y en diferentes ocasiones, lo verdaderamente recordado en nuestra vida pública es el desprecio", escribe Nugent en El laberinto de la choledad (1992; p. 21). "despreciamos y somos despreciados de muchas maneras", continúa el autor, para concluir: "lo que importa es que ese desprecio no pase desapercibido".

En la presente comunicación se informa acerca de un estudio acerca de la presencia de este afecto en la sociedad peruana.

\section{El desprecio en el Perú}

Borrar al otro del grupo de actores de nuestro escenario vital: así define al desprecio el sutil fenomenólogo alemán Philip Lersch (1968). En su libro La estructura de la personalidad. Lersch señala que tanto la estima del otro como el desprecio son emociones de la convivencia. Si despreciamos a alguien no queremos convivir con él; no queremos que nuestra vida se cruce con la suya.

Pero ¿cuán cierta es la afirmación de Nugent? $Y$, si es verdad que el desprecio es moneda de uso diario en el Perú, ¿cómo lo sienten y lo viven los peruanos, los que desprecian así como los que son despreciados?

Pocos que conozcan nuestra sociedad y que estén inmersos en el tráfago de ella pondrán en tela de juicio la afirmación de Nugent. Sin embargo, como muchas otras de índole semejante y siempre de carácter negativo acerca de la calidad de las relaciones sociales en el Perú (tales como, por ejemplo, "en el Perú hay mucha envidia", "en el Perú hay mucha hipocresía", "en el Perú hay mucho resentimiento"; "la gente en el Perú es muy rajona", etc.), ella puede ser entendida como un cuadro impresionista, una visión lúcida de la realidad peruana que, sin embargo, está sólo basada en observaciones agudas pero no sistematizadas. 
Es claro que el aserto de Nugent sólo puede ser plenamente comprobado por medio de la observación directa de los actores sociales en la vida cotidiana. El desprecio es un afecto extremo, uno de los sentimientoslimite que se puede experimentar hacia otra persona, que no sólo se expresa a través de palabras sino que, más bien, se concreta en conductas y en acciones (o tambien en la ausencia de estas últimas).

Es así que el desprecio en el Perú puede rastrearse por ejemplo en una conversación espontánea y en testimonios personales, como también en la literatura. Cuestión de piel. Testimonios de racismo en el Perú (1993) presenta conmovedores ejemplos; y, Mario Vargas Llosa lo identifica en la obra de Arguedas, como lo certifica la siguiente cita tomada de La utopía arcaica (1996): "El racismo es omnipresente en la sociedad abanquina: los blancos desprecian a los indios y a los mestizos, y los mestizos desprecian a los indios y alientan un sordo resentimiento contra los blancos. Y todos ellos -blancos, indios y mestizos- desprecian a los negros. Una de las más dramáticas escenas de la novela [Los ríos profundos; $\mathrm{N}$. de los A.] -uno de sus cráteres- ocurre cuando Lleras prefiere escaparse del colegio antes que pedir perdón al hermano Miguel, por el asco que le merece el color de su piel: !No! !Es negro, padrecito! !Es negro! !Atatauya! (p. 103). El racismo contamina inconscientemente al propio Ernesto, quien se pregunta asombrado: "¿Cómo, siendo negro, el Hermano pronunciaba con tanta perfección las palabras? ¿Siendo negro?” (p. 104). Por su parte, las señoras de Abancay, cuando se apiadan del hermano golpeado por Lleras, aclaran que su compasión se debe a que se trata de un religioso, condición que a sus ojos lo redime del baldón de su raza: "Aunque sea negro, tiene hábitos", dicen (p. 116)".

Un rígido sentido de la jerarquia social, basado no en méritos objetivos ni en logros personales sino en un conjunto de inmodificables supuestos acerca del valor de los seres humanos que ponen de manifiesto la todavía existente ( $\mathrm{y}$ omnipresente) herencia colonial en la sociedad peruana: el autoritarismo; $y$, por último, el racismo tempranamente instilado en la mentalidad de los peruanos (Flores Galindo, 1994; Portocarrero 1993) dan fuerza a la proclividad al desprecio y a la discriminación que 
se observa aquí. Constituyen poderosos obstáculos en el camino que nuestro país debe recorrer para conformar una sociedad moderna, siempre sinónimo de una sociedad igualitaria.

En el sentido de la jerarquía al que nos hemos referido, sexo y raza juegan un papel de primera importancia. Patricia Oliart los comenta: "Al construir nociones de jerarquía social asociadas con aspectos biológicos -como son el sexo y la raza- dichas jerarquías fueron naturalizadas y presentadas como eternas, incluso en una era en la que las ideas meritocráticas y democráticas eran ampliamente divulgadas. De este modo, las personas de clases medias y bajas confrontaban diariamente un conjunto de ideas destinadas a formar su autopercepción como seres indignos de mejorar su lugar en la escala social debido a su género o raza. Una persona oscura era colocada en la escala social de acuerdo a su grado de blancura y su género" (sin fecha, p. 85).

Esto que aún pervive, fue una práctica habitual durante las primeras décadas de este siglo. "Hasta 1930, más o menos, existía un veto en algunas familias para quienes no tenían otra credencial que su dinero", afirma Jorge Basadre, citado por Burga \& Flores Galindo (1984), quienes a su vez agregan: "aunque los orígenes de las familias oligárquicas, en la mayoría de los casos, se remontaban apenas a la época del guano, la pertenencia de la clase se definía además por el apellido, lazos de parentesco, cierto estilo de vida; en otras palabras, a lo que sería por criterios estrictos de 'clase' se añadían otros de tipo 'estamental', como rezago y herencia de la colonia" (p. 84).

De otro lado, el acendrado autoritarismo, que muchas veces asume el rostro (un poco más humano) de paternalismo, atenta contra el desarrollo de una democracia sólida. La crítica y la autocrítica; la discusión de las ideas en la búsqueda del consenso; la equidad; la alternancia, y, si fuera necesario, la revocación de la autoridad; el respeto por la opinión ajena, todos estos rasgos característicos de una sociedad democrática y, al mismo tiempo, fortalecedores de la autoestima y de la sensación del propio valor de cada ciudadano, faltan entre nosotros. En su lugar están 
el autoritarismo y el servilismo: la adulación y el desacuerdo que sólo se masculla o se murmura; la altanería y el desdén.

Por último, el racismo soterrado en la sociedad peruana, da fuerza al sentimiento de "que no somos iguales", de que unos valen más que otros, y sirve así de sustento a un orden social de escasa movilidad y al autoritarismo.

No se trata, por cierto, de que cada uno de estos rasgos actúe de modo independiente: ellos se encuentran vinculados entre sí, reforzándose mutuamente en una mentalidad que aún hoy se revela impermeable al cambio de algunas de sus más retrógradas actitudes.

Esta constelación de elementos ha hecho del estado peruano una realidad discriminadora y segregacionista, "controlado por una minoría social heredera de los privilegios de los conquistadores y que se sentía profundamente ajena y separada de la mayoría de la población, de la cual la separaba no sólo la lengua y las costumbres sino ante todo los prejuicios racistas de cuño colonial, que provocaron que a lo largo del siglo XIX la élite criolla identificara proyecto nacional con política de inmigración (europea), pues ideológicamente se consideraba que el Perú era un "país vacío" - los indios no eran peruanos- que tenía que ser poblado por colonos de raza blanca, que permitieran explotar sus riquezas naturales" (Manrique, 1994; p. 237).

Pero, por supuesto, no sólo el Estado; también y sobre todo la sociedad, tal como se puede observar en la vida cotidiana en las ciudades, en las que hay una organización jerárquica y, por ende, una estratificación; esto es, una ciudad "en la que sus habitantes evitan conscientemente encontrarse" (Adrianzén, 1990; p. 29). Potestá y La Rosa (1996) encontraron que universitarios que vivían en el estrato A de Lima (La Molina, San Borja, San Isidro) respondian mayoritariamente afirmando desconocer zonas de clase baja, como El Centro, Rímac, La Victoria, Comas, San Martín de Porras, Breña, etc. (para información histórica sobre el particular puede consultarse Del Aguila Peralta, 1997; y, Ortega, 1986). 


\section{¿Cómo evaluar el desprecio?}

Volvamos, sin embargo, a la tajante afirmación de Nugent. ¿Cómo comprobarla?

El término desprecio es muy duro, y el peruano común y promedio muestra una cierta angustia frente al empleo de palabras definitivas, prefiriendo más bien la práctica del buen decir, la expresión eufemística. Tal vez por ello Kogan (1996) señala que el término más apropiado es frialdad.

Sea como fuere, desprecio o frialdad son, como ya se dijo, fenómenos afectivos que pueden verificarse y evaluarse sobre todo a través de la observación o de la obtención de testimonios, que, lamentablemente, suponen procedimientos costosos en recursos humanos y tiempo. Una excepción la constituye, sin embargo, el reciente trabajo de Evans (1996), que ha estudiado el desprecio entre estudiantes universitarios limeños a partir del análisis de graffiti.

Además de estas posibilidades están también los procedimientos de autoreporte, tales como cuestionarios, inventarios, y encuestas, entre tanto de difundido empleo en la investigación social.

Como se sabe, las técnicas que recurren al autoreporte están limitadas por una serie de posibilidades de error, que van desde el premeditado y activo falseamiento de las respuestas hasta la influencia de fenómenos tales como la aquiescencia y la deseabilidad social (véase Furnham 1986; de utilidad es asimismo Tuesta Soldevilla 1997). Sin embargo, ante la relativa imposibilidad de la observación directa sistemática, son recursos que no pueden ser desdeñados a pesar de sus limitaciones.

Resulta claro que la afirmación de Nugent constituye un sombrio diagnóstico de la realidad psicosocial peruana. Como ya dijimos, el sostener que en el Perú hay mucho desprecio no es la única apreciación negativa de la realidad social de nuestro país. También están la creencia de que la gente es hipócrita y de que hay mucha envidia entre nosotros. 
Cabe preguntarse por tanto por qué no han sido objeto de investigaciones.

Se trata, como bien lo sabemos, de verbalizaciones que por lo general se escuchan en conversaciones informales: en el decurso de ponencias académicas como una afirmación incidental; o, como apreciaciones concluyentes en el relato de situaciones interpersonales desagradables. Esta característica de ser una información casi accesoria, que aparece en medio de una conversación o en una discusión; este rasgo casi de anécdota que muchas veces tienen, han determinado el escaso interés de los estudiosos de la psicología y la sociología.

Hoy, sin embargo, la vida cotidiana y los afectos se han convertido en una dimensión de imprescindible estudio en el análisis de la realidad social, abriendo las puertas al mejor conocimiento de las mentalidades (Barrig y Henríquez sin fecha; p. XIII).

Por eso es que creemos de interés complementar las apreciaciones acerca de la calidad de la vida social en nuestro país con datos cuantitativos. Ellos contribuyen a distinguir si los fenómenos que observamos son hechos fortuitos, aislados, vinculados quizás sólo a las peculiaridades de alguna persona; $o$, si, más bien, estamos ante tendencias y modos de ver y sentir la realidad social que, aunque no claramente verbalizados, igual determinan la conducta de los peruanos. Esta fue la razón para llevar a cabo este trabajo.

\section{Métodología}

Se utilizó un conjunto de seis afirmaciones tomadas de una encuesta de dimensión mayor acerca de actitudes raciales y estereotipos regionales en estudiantes universitarios (León 1998). Dichas afirmaciones conforman la Fiscala de Percepción de Desprecio en el Peru, cuyo marco referencial y proceso de validación se describe en el anexo.

Los participantes fueron 789 estudiantes de dos universidades, una particular y una nacional. De ellos 362 fueron hombres y 427 fueron 
mujeres, entre 15 y 30 años de edad). Respondieron a la escala como parte de la encuesta mayor a la que hemos hecho referencia previa.

Se les pidió además que respondieran una breve encuesta en la que se les solicitaba que valoraban el "porcentaje" que ellas creían tener de blanco, negro, andino y oriental, de acuerdo con las siguientes posibilidades: "nada", "muy poco", "algo", "mucho" y "99-100\%".

\section{Resultados}

En el proceso de obtención de los resultados se siguieron varios pasos. En primer lugar, y con el propósito de extraer la mayor cantidad de información sobre un tema que, como el desprecio, es de tan difícil acceso, se procedió a analizar las respuestas a cada uno de los items. Este análisis se llevó a cabo a través de la determinación de la frecuencia de respuestas por sexo para cada una de las cinco posibilidades que consideró el reactivo elaborado.

Seguidamente, se calcularon los puntajes promedio por sexo para toda la escala y se procedió a la averiguación de la presencia de diferencias significativas mediante la $\mathrm{t}$ de Student.

Dado que el empleo de esta técnica exige el cumplimiento de dos requisitos centrales, el de la normalidad de la distribución y el de la homogeneidad de las varianzas, se procedió al cálculo de ambas, cuyos resultados se presentan en sendos Cuadros.

Con posterioridad, se llevaron a cabo otros análisis que son asimismo reportados en esta comunicación.

En cuanto al análisis de items, en el Cuadro 1 se presenta los porcentajes de respuestas por sexo para cada uno de los ítems.

Porcentajes elevados de hombres $(85.9 \%$ ) y mujeres $(88.6 \%)$ reportan haber observado que alguien era discriminado (ítem 2) y sólo pocos negaron tal observación. 


\section{Cuadro 1}

Porcentajes de Respuestas de la Escala de Percepción de Desprecio en el Perí según Sexo

\begin{tabular}{|c|c|c|c|c|c|c|c|c|c|c|c|c|c|c|}
\hline & $N$ & & I & $\mathrm{F}$ & & & & IS & $B C$ & & $\pi$ & & $\begin{array}{c}\text { Y } \\
\text { Commet }\end{array}$ & $p$ \\
\hline Item & $\mathrm{H}$ & & $1 \mathrm{H}$ & M & $\mathrm{H}$ & M & $\mathrm{H}$ & $\mathrm{M}$ & $\mathrm{H}$ & M & $\mathrm{H}$ & M & & \\
\hline 01 & 0.8 & 0.5 & 15.6 & 18.7 & 15.1 & 12.6 & 12.5 & 7.7 & 38.3 & 42.9 & 17.7 & 17.6 & .096 & .205 \\
\hline 02 & 1.4 & 1.4 & 2.2 & 2.8 & 4.4 & 4.2 & 6.1 & 3 & 39.8 & 38.9 & 46.1 & 49.7 & .079 & .428 \\
\hline 03 & 1.1 & 0.2 & & 3.5 & 11.6 & 7 & 10.7 & 10.5 & 53.9 & 54.1 & 18.8 & 24.7 & .112 & .077 \\
\hline 04 & 0.8 & 0 & 4.1 & 1.4 & 8.8 & 6.1 & 12.6 & 8.9 & 54.1 & 61.6 & 19.6 & 22 & .142 & $.007^{*}$ \\
\hline 05 & 0.6 & 0.7 & 4.1 & 2.3 & 5.8 & 5.6 & 11.9 & 5.4 & 55.2 & 56.7 & 22.4 & 29.3 & .141 & $.008^{*}$ \\
\hline 06 & 0.3 & 0 & 7.5 & 11.5 & 9.4 & 11 & 11.3 & 5.5 & 40.3 & 44.1 & 31.2 & 27.9 & .142 & $.014^{*}$ \\
\hline
\end{tabular}

${ }^{*} \mathrm{p}<0.05$

NR: No responde

TF: Totalmente falso

BF: Bastante falso

NS: No sć

BC: Bastante cicrto

TC: Totalmente cierto

Hombres y mujeres, pero sobre todo ellas, son del parecer que en el Perú la gente blanca "basurea" a las de otras razas (item 4) y que ticne ventajas sociales (ítem 5). En el caso del ítem 4, la percepción de desprecio ("basureo") es mucho mayor entre las personas del sexo femenino (83.6 \%) que entre los varones, entre quienes sin embargo también es elevada $(73.7 \% ; p<0.05)$; lo mismo y en porcentajes relativamente similares ocurre en el ítem 5, "siento que en determinados contextos las personas blancas tienen acceso a mejores oportunidades de vida" $(\mathrm{H}: 77.6 \% \mathrm{y}$ M: $86 \% ; p<0.05)$.

Aunque hay relativa unanimidad de opiniones acerca de lo cierto de la afirmación contenida en el item 6 , "cuando en un aviso se solicita "buena presencia' me parece que el color de la piel de quien se presenta juega un rol importante", pues el $71.5 \%$ de varones y el $72 \%$ de las mujeres 
la consideran bastante o totalmente cierta, llama la atención el hecho de que son las mujeres las que en mayor porcentaje la consideran falsa $(\mathrm{H}$ : $16.9 \%$ y M: $22.5 \% ; p<0.05)$.

El ítem que explora de modo más decidido la percepción de desprecio en el Perú, el número 3 ("aca la gente es muy despreciativa") arroja resultados parecidos en uno y otro sexo, si bien son las mujeres la que están un poco más de acuerdo con él: $72.7 \%$ de varones $v s 78.8 \%$ de mujeres.

De singular dramatismo nos parecen los resultados en el item 01 , "entre mis amigos hay personas que desprecian o tratan mal a personas negras o de los andes": $56 \%$ de varones y $60.5 \%$ de mujeres.

A continuación se analizará la percepción de desprecio por autoasignación racial y sexo. Con el propósito de evaluar si hay diferencias en la percepción de desprecio entre las categorías de cada tipo de autoasignación racial se utilizó la prueba $F$ en un análisis de varianza de una vía (ANOVA). Previamente, se verificaron los supuestos de dicha prueba: normalidad de frecuencia y homogeneidad de varianza.

A fin de evaluar la normalidad se utilizó la prueba de KolmogorovSmirnov, que usa una prueba $Z$. La significancia a dos colas, o valor $p$, es la probabilidad de obtener el valor $Z$ o un valor más extremo; es decir la probabilidad de rechazar la normalidad. El Cuadro 2 muestra los valores obtenidos.

Como únicamente se encuentran tres valores de significancia $p<0.01$ en el Cuadro anterior, se concluye que hay igual número de grupos en los que no hay normalidad, el sexo femenino y la autoasignación de raza oriental en términos de ausencia total de ella ( $\mathrm{Nada}$ ); y la autoasignación de raza blanca en términos de Algo. En todos los otros casos, se da por establecida la normalidad de la distribución de puntajes de desprecio.

Sin embargo, todos los valores $p$ son mayores que 0.0001 (valor minimo), por lo que se puede afirmar que para un nivel de significación 


\section{Cuadro 2}

Prweba de Normalidad para el Constructo de Percepción del Desprecio en el Perí

\begin{tabular}{|lrcccc|}
\hline Grupo & $\mathbf{n}$ & Media & DE & $\boldsymbol{Z}$ & $\boldsymbol{P}$ \\
\hline Sexo Masculino & 350 & 22.71 & 3.98 & 1.46 & .028 \\
Sexo Femenino & 415 & 23.14 & 3.80 & 2.18 & $.001 *$ \\
\hline Raza Andina & & & & & \\
Nada & 165 & 22.67 & 4.45 & 1.16 & .138 \\
Muy poco & 258 & 22.78 & 3.78 & 1.25 & .087 \\
Algo & 239 & 23.16 & 3.50 & 1.43 & .034 \\
Mucho & 37 & 23.62 & 3.10 & 0.64 & .809 \\
$99-100 \%$ & 3 & 24.33 & 5.51 & 0.33 & .500 \\
\hline Raza Blanca & & & & & \\
Nada & 43 & 22.95 & 4.59 & 0.58 & .887 \\
Muy poco & 166 & 22.44 & 4.41 & 1.43 & .034 \\
Algo & 419 & 23.03 & 3.64 & 1.97 & .001 \\
Mucho & 102 & 23.02 & 3.73 & 1.07 & .204 \\
$99-100 \%$ & 10 & 26.20 & 2.90 & 0.55 & .927 \\
\hline Raza Negra & & & & & \\
Nada & 306 & 23.22 & 3.82 & 1.48 & .025 \\
Muy poco & 241 & 22.56 & 4.07 & 1.44 & .032 \\
Algo & 124 & 22.93 & 3.52 & 1.01 & .259 \\
Mucho & 12 & 22.67 & 3.42 & 0.77 & .693 \\
\hline Raza Oriental & & & & & \\
Nada & 459 & 22.85 & 3.91 & 1.93 & $.001 *$ \\
Muy poco & 100 & 23.21 & 3.74 & 0.94 & .343 \\
Algo & 23.03 & 3.71 & 1.27 & .081 \\
Mucho & 21.80 & 2.95 & 0.86 & .445 \\
$99-100 \%$ & 25.50 & 3.51 & 0.52 & .447 \\
\hline
\end{tabular}

Nota. $* p<0.01$. Hay 23 casos en los que no se obtuvo respuesta en el constructo Percepción de Desprecio en el Perú.

menor de $0.01 \%$ (como ya se dijo, sólo en dos casos el valor $p$ es menor de $1 \%$ ) se rechazaría la hipótesis de normalidad. Por ello, la significación con un $99.99 \%$ de confianza permite aceptar la hipótesis de normalidad en todos los casos. Cabe aquí una precisión de corte técnico: cuando el 58 
tamaño de la muestra es grande (como en este caso), siempre cualquier prueba de bondad de ajuste puede llevar al rechazo de la hipótesis nula. Es siempre imposible encontrar datos que sean exactamente distribuidos en forma normal.

La segunda condición es la de la homogenidad de varianzas, evaluada por medio del test de Levene. Este test calcula para cada caso la diferencia absoluta entre el valor para cada caso y su promedio de celda y luego ejecuta un ANOVA de una vía de esas diferencias. Para tamaños de muestras grandes, un valor $\mathrm{p}$ no significativo significa que existe evidencia insuficiente que las varianzas difieren. El test de Levene es sensitivo a la falta de normalidad. El Cuadro presenta la prueba de homogeneidad de varianzas de las áreas evaluadas.

Se observa que hay homogeneidad de varianzas en todos los casos menos en Raza Andina. Todos los valores p son mayores que 0.008 (valor mínimo salvo en caso de la Raza Blanca). Por ello, con un $99.2 \%$ de confianza se acepta la hipótesis de homogeneidad de varianza en todos los casos.

Los promedios para toda la escala fueron de 22.70 (DS: 3.97 ) en el sexo masculino; y de 23.13 (DS: 3.79), en el sexo femenino; con una $t$ de -1.53 , no significativa. Esto también se puede ver en el Análisis de varianza.

\section{Cuadro 3}

Prueba de Homogeneidad de L'arianzas de las áreas evaluadas

\begin{tabular}{|lcccc|}
\hline VARIABLES & gl 1 & gl 2 & Test de Levene & $p$ \\
\hline Sexo & 1 & 763 & 1.37 & .243 \\
Raza Andina & 4 & 697 & 3.50 & $.008 *$ \\
Raza Blanca & 4 & 735 & 2.35 & .053 \\
Raza Negra & 3 & 679 & 0.67 & .573 \\
Raza Oriental & 4 & 674 & 0.72 & .579 \\
\hline
\end{tabular}

$* p<0.01$ 


\section{Cuadro 4}

Análisis de Varianza de una sola Via Autoasignación Racial y Discriminación

\begin{tabular}{|c|c|c|c|c|c|}
\hline & g) & $\begin{array}{l}\text { Suma de } \\
\text { cuadrados }\end{array}$ & $\begin{array}{l}\text { Promedio de } \\
\text { cuadrados }\end{array}$ & $F$ & $p$ \\
\hline \multicolumn{6}{|l|}{ Raza Andina } \\
\hline Entre grupos & 4 & 53.06 & 13.27 & .90 & .462 \\
\hline Intra grupos & 697 & 10242.18 & 14.69 & & \\
\hline Total & 701 & 10295.24 & & & \\
\hline \multicolumn{6}{|l|}{ Raza Blanca } \\
\hline Entre grupos & 4 & 152.12 & 38.03 & 2.52 & $.040 *$ \\
\hline Intra grupos & 735 & 11099.90 & 15.10 & & \\
\hline Total & 739 & 11252.01 & & & \\
\hline \multicolumn{6}{|l|}{ Raza Negra } \\
\hline Entre grupos & 3 & 59.91 & 19.97 & 1.35 & .259 \\
\hline Intra grupos & 679 & 10077.28 & 14.84 & & \\
\hline Total & 682 & 10137.19 & & & \\
\hline \multicolumn{6}{|l|}{ Raza Oriental } \\
\hline Entre grupos & 4 & 63.42 & 15.86 & 1.08 & .366 \\
\hline Intra grupos & 674 & 9901.62 & 14.69 & & \\
\hline Total & 678 & 9365.05 & & & \\
\hline
\end{tabular}

$* p<0.05$

A partir de los resultados previos se decidió averiguar las relaciones entre la autoasignación racial y los puntajes de discriminación. Esto se hizo a través de un ANOVA, cuyo resumen se presenta en el Cuadro 4.

El ANOVA permite afirmar que en las autoasignaciones raciales andina, oriental y negra no hay diferencias en discriminación en cada una de sus categorias, pero que si las hay en la autoasignación racial blanca $(F=2.51 p<0.05)$.

Por ese motivo se decidió averiguar cómo se producían tales diferencias al interior de la autoaveriguación racial blanca. Los resultados se dan a conocer en el Gráfico 1. 
Humillados y ofendidos

\section{Gráfico 1}

Promedios en la Escala de Percepción de desprecio en el Peri por las diferentes categorias de Autoasignación Racial Blanca (ARB)

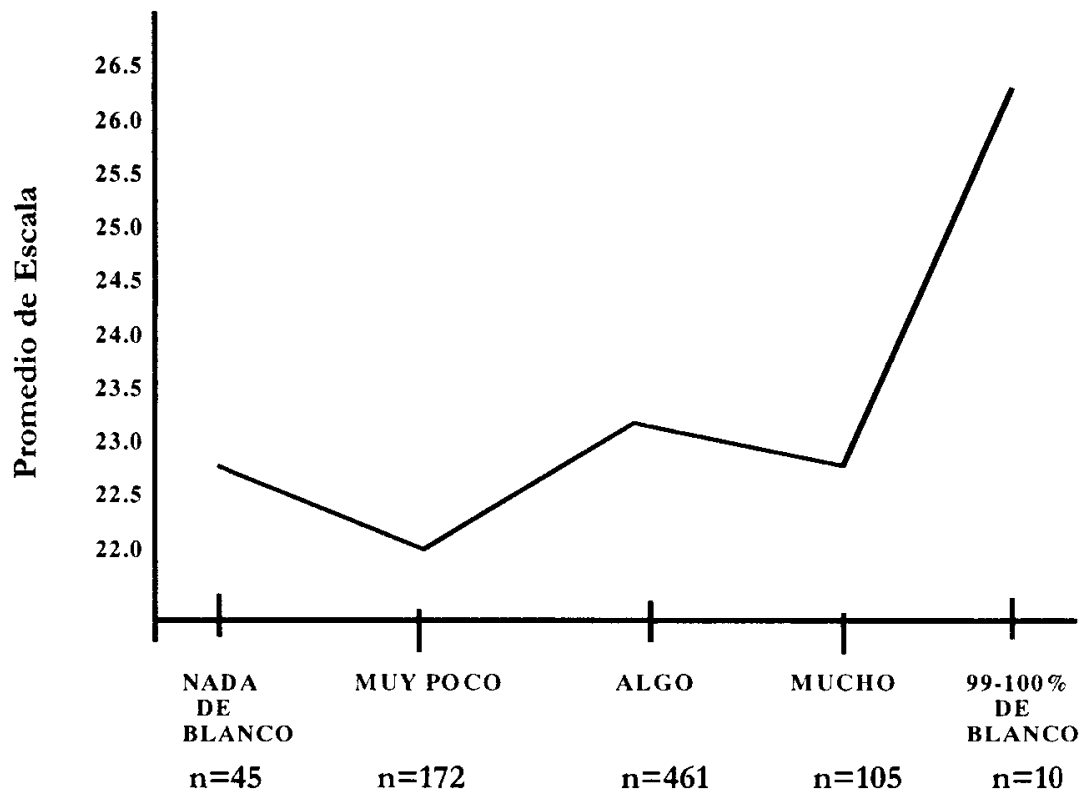

Categoria ARB

Bajo el Test de Rasgos Múltiples LSD las cuatro primeras categorias son similares en cuanto a su promedio de desprecio y muy por debajo del promedio de desprecio es la categoría 99-100\% de blanco. Puede decirse, como conclusión, que a mayor autoasignación racial blanca (99. $100 \%$ ) mayor percepción de desprecio en el Perú.

\section{Discusión}

Como ya se dijo, sentimientos como el desprecio pueden ser adecuadamente valorados sólo a través de una indagación detenida de la 
subjetividad de quienes los sienten y del que se cree víctima de ellos. Las encuestas ofrecen únicamente información aproximada. Es, por tanto, con la debida cautela con la cual habremos de comentar los resultados obtenidos.

Impresiona, en primer lugar, la unanimidad de hombres y mujeres con respecto al ítem 3, "acá la gente es muy despreciativa".

Estos resultados son una constatación numérica de la afirmación de Nugent (1992) y confirman los que reportaron Dejo Soto (1992) y Twanama (1994), quienes han investigado la discriminación racial en Lima. Los hallazgos de Dejo Soto y Twanama, junto con los nuestros, más la apreciación de Nugent, permiten afirmar que la sensación subjetiva de ser discriminado es muy frecuente entre los peruanos. Todos los espacios sociales (la escuela, el trabajo, las oficinas públicas, la calle misma), constituyen el escenario para la discriminación (Dejo Soto 1992), que revela el desprecio que subyace a ellas.

Cabe aquí anotar, como lo ha destacado Twanama (1994), que la discriminación tiene un carácter opaco: las personas se sienten discriminadas pero no precisan por qué; cuál es la causa de esa discriminación. Inclusive, podríamos aventurar que los mismos discriminadores no tienen muy en claro qué es lo que están discriminando, ni tampoco las causas por las cuales discriminan.

En los últimos años el reconocimiento de un fenómeno psicosocial tan difundido en el Perú como es el racismo ha echado luces acerca de las causas y la naturaleza de la discriminación y el desprecio en el Perú.

Como lo han señalado estudiosos del racismo en el Perú (e.g. Portocarrero 1992, 1993; Flores Galindo 1994) no se trata sólo de prejuicios referidos a la raza en un país como el nuestro, en el cual la inmensa mayoría se sabe y se siente mestiza. Los prejuicios en el Perú son étnico-sociales, y recorren todos los estratos sociales en el país.

El origen de ellos se remonta como mínimo al siglo XVIII. Cecilia Méndez señala que "la ecuación indio-bestia no fue ciertamente una invención de la República. La popularización de esta conceptualización 62 
en el imaginario de las elites parece estar vinculada más bien, junto con la influencia de las ideas de la ilustración, a los cambios producidos en la sociedad andina posteriores a la rebelión de Túpac Amaru" (1997; p. $20)$.

Pero como en el Perú todos tenemos "un poco de todo", discriminar a alguien sólo por el color de su piel o por la supuesta raza a la cual él pertenece, sería algo atentatorio contra un aspecto de nosotros mismos.

Dado que en el país hay todavía una cercana relación entre color de la piel y clase social, nuestros prejuicios se dirigen a aquellos que por uno $\mathrm{u}$ otro factor se encuentran en situación de inferioridad. Es decir, la discriminación tiene como víctimas a quienes son de "raza inferior" $y$ además pertenecen a los niveles sociales más bajos.

En La utopia arcaica Mario Vargas Llosa (1996) expone este fenómeno señalando que “el concepto de 'blanco' en el contexto peruano -como en todos los países andinos-, es racial al mismo tiempo que económico, $y$, en muchos casos, este último factor es el determinante de la supuesta clasificación étnica. Quien asciende en la escala económica se va blanqueando, en tanto que el que se empobrece se indianiza, achola o ennegrece a los ojos de los demás" (p. 166).

No es difícil imaginar las consecuencias para la sociedad peruana de la difundida sensación de desprecio que los peruanos experimentan. Como afecto social, el desprecio y su expresión más abierta, la discriminación, constituyen una severa valla para el desarrollo de sentimientos de solidaridad, decisivos en la forja de cualquier nación; y, de esencial igualdad, definitorios de toda sociedad que con derecho de autodefina como democrática.

Desprecio y discriminación sentidos y expresados hacia una persona provocan en ella sentimientos no menos negativos que, como es lógico, hacen lo suyo para impedir un minimum de empatía entre los individuos que conforman una sociedad. Este desencuentro constituye una de las tragedias del Perú y es la base para el escepticismo, el pesimismo y la 
impotencia que "parecen haber traspasado los propios tuétanos de la sociedad peruana" (López Jiménez 1994; p. 24). Pero, además, el desprecio $y$ la discriminación provocan un sentimiento al que se refiere Manrique (1992), apenas explorado en el Perú de hoy; el de la humillación.

$\mathrm{Si}$ la humildad es "la actitud en que el individuo se conduce respecto a otras personas como si fuera inferior a ellas" (Warren 1963; p. 167), la humillación puede ser entendida como el sentimiento de profunda agresión a la autoestima que surge en una situación en la que el humillado es públicamente maltratado, sin consideración alguna a su imagen social, de modo tal que su valor como persona es reducido al mínimo posible; $o$, cuando sus méritos y capacidades son abiertamente subestimados $\sin$ que siquiera se le dé la oportunidad de evidenciarlos.

Vivencias de todos los días en el Perú de hoy y de siempre. Vivencias que se dan, para sólo mencionar un ejemplo, en "esos jóvenes provincianos que logran acceso a la escuela, estudian difícilmente en el convencimiento de que la educación es la vía para ser admitidos por el país oficial -tener empleo, ser tratados con respeto, mirados de igual a igual-, llegan así a las puertas de las universidades y, en plena juventud, descubren que nada de eso es posible en un país de desempleados y donde además existen otras reglas, no escritas ni admitidas, pero efectivas: descubren el autoritarismo y el racismo" (Flores Galindo 1988; p. 25).

Vivencias, por último, que no dejan de traer consecuencias en el plano social. Escribe Harré: "algunos estudios sobre la adolescencia han mostrado cómo muchos jóvenes tienen un interés y preocupación casi obsesivos por el mantenimiento de la dignidad, examinando cuidadosamente el ambiente social en busca de ocasiones y actos de humillación posibles. Cuando se han identificado tales actos, algunos adolescentes pueden practicar la venganza violenta [...]" (1982; p. 42).

Los que se sienten "más" maltratan a los que son "menos", respaldados por una cultura que experimenta una extraña fascinación por el autoritarismo practicado por uno mismo o que se ejerce sobre uno. 
Hagamos una digresión para señalar que, ciertamente, hay quienes se autohumillan. Lo hacen en busca de algún beneficio (Delgado, 1974) o, sencillamente, como garantía para prevenir la agresión de los poderosos, en una sociedad en la cual el rencor $y$ la revancha siempre han estado presentes (Gonzáles 1996). Se trata de una forma perversa de virtualizar el consejo que daba San Ignacio de Loyola a los jesuitas: "se debe ganar a los doctores de la Universidad y a las personas de autoridad con humildad, modestia y buenos oficios" (1982; p. 782). Humildad, modestia y buenos oficios pueden ser remplazados por fenómenos familiares para nosotros: servilismo, obsecuencia, oportunismo.

Los resultados a varios de los items permiten obtener alguna idea acerca de por qué los encuestados afirman que acá la gente es muy despreciativa. Son muchos los que afirman haber sido testigos de actos de discriminación dirigidos a otras personas, básicamente por su raza (items 2 y 4). Los escenarios: como ya lo dijimos, la calle, las oficinas; las opiniones formuladas en el seno de la familia acerca de alguna persona; el círculo de amigos. Es decir: todas las esferas de la vida social en el Perú.

En todas las sociedades en las cuales hay crasas diferencias de clase el desprecio es una realidad. El Perú no es la excepción. La pobreza en el Perú, como en todas partes, supone menor nivel instruccional, menores habilidades verbales: menor acceso a servicios y, en una sociedad inequitativa como es la nuestra, menor capacidad para hacer valer los derechos cuando son violados. Los pobres tienen una ciudadanía limitada en el Perú (López 1996, 1997).

Reynaldo Alarcón caracteriza del modo siguiente a los pobres en países como el nuestro: "Aquí los pobres tienen visibilidad social, se les detecta a distancia sin otro dato que su aspecto exterior: ropas raídas, faltos de higiene, físicamente fatigados y de poco peso, sin hábitos burgueses. El barrio pobre y la casa de vecindad, sea el tugurio del callejón o la vivienda de la barriada, se imponen a la vista y al olfato. Tienen olor: huelen a desagüe, a humo de kerosene, a seres humanos aglomerados, a aire 
contaminado, a humedad. La pobreza no está escondida, la tenemos a paso, presente: a diferencia de lo que, según Harrington, ocurre en las sociedades desarrolladas. Es, justamente, a partir del aspecto exterior que la gente de los sectores medios y altos juzgan a los pobres" (1986; p. 108; el subrayado es nuestro).

Es por eso cierta la aseveración de Callirgos al señalar que "la asociación pobreza -choledad-suciedad es repetida en los mensajes de los sectores privilegiados. Una manera de afirmar la supuesta 'blancura' propia. O una manera de condenar a los pobres "porque son sucios"” (1997; p. 37).

Ese juicio negativo acerca de los pobres, en quienes se reconoce a personas con menores ingresos, con menor instrucción, con menores posibilidades de desarrollo individual; en fin, a seres diferentes, también por lo general en el color de la piel ("negros", "zambos", "indios"), cxplica la tendencia a "darse su lado" de muchas de las personas de la clase alta y media.

Fs una perogrullada decir que la pobreza en el Perú asume perfiles dramáticos. Ella abarca a la inmensa mayoría de la población (Eguren et al. 1997) pero, además, alcanza una magnitud alarmante en su forma más injusta y dolorosa: la miseria.

$\mathrm{Y}$, sin embargo, la carta política que nos rige y todas las que nos han regido, declaran que en el Perú todos somos iguales. Pero esa igualdad es sólo nominal, ya que sabemos -como lo escribe Flores Galindo (1988)"que tanto en las decisiones importantes como en la vida cotidiana, no todas las opiniones tienen el mismo valor: depende de quien las diga, cuál sea su aspecto físico, cómo se apellide, quiénes sean sus parientes y, desde luego, en qué nivel socioeconómico se ubique" (p. 24).

La igualdad es sólo en el papel y nada más. Ni siquiera ha calado en nuestro modo de vernos y de entendernos, pues "en el Perú hay conciencia de igualdad pero no hay un sentimiento fuerte de igualdad" (Portocarrero 1992; p. 187).

66 
Blancos y cholos: ricos y pobres; costeños y serranos; éstas son sólo algunas de las diferencias que hacen que la sociedad peruana esté profundamente escindida y que en ella sentimientos tales como el desprecio, la discriminación y la humillación sean tan frecuentes.

Son las mujeres las que en nuestro estudio tienen una percepción más aguda del desprecio en el Perú. Esta percepción más negativa guarda concordancia con otros resultados obtenidos por nosotros, en los que de modo sistemático se observa que las mujeres (nos referimos en particular a mujeres adolescentes y jóvenes de lo que grosso modo podemos llamar clase media), consideran, en proporción mayor que los varones, que en el Perú hay mucha envidia (León y Moscoso 1991; León y Martell 1994); y que la gente es muy hipócrita (León et al., en preparación). Esto probablemente juega algún papel en el reporte que las mujeres hacen (en proporción mayor que los hombres) de estar tensas (León y Romero 1988; León y Sirlopú 1996) y de experimentar mayor ansiedad social (León y Portocarrero 1989; León y Tejada 1996).

Propongamos una explicación para esto. Los procesos de socialización de hombres y de mujeres son en un país como el nuestro muy diferentes. Si bien en los últimos años tales diferencias van tornándose menores, subsisten aún patrones distintos. Los hombres se educan para demostrar fuerza y poder. "Son los varones quienes se esfuerzan más en manejar símbolos de poder y prestigio. El modelo ideal de joven es el agresivo, abusivo, diestro en los deportes, el que descuida sus estudios y desafia a las autoridades del plantel, el que viste y peina a la moda. Se ven conminados a seguir un modelo que fomenta la violencia y la competitividad entre los pares, lo cual, en la práctica, significa demostrar hombría en todo momento", escribe Callirgos (1996; p. 50).

Norma Fuller, autora de dos interesantes estudios acerca de la identidad femenina (Fuller 1993) y masculina (Fuller, 1997), ha tratado igualmente el tema de la socialización diferencial. Cedámosle la palabra: "El lenguaje del fútbol, plagado de referencias al dominio, control y posesión de las mujeres y al rechazo a la sexualidad homosexual pasiva, produce 
y reproduce la cultura masculina. Los varones se afilian a uno u otro equipo de fútbol independientemente de su origen racial étnico o de clase; ello los entrena en el trato con personas de diferente origen socio-étnicoracial y les proporciona un lenguaje y un código de conducta que borra las jerarquías sociales y hace de la calle el espacio del encuentro y la igualdad. De este modo, la participación en la institución deportiva y del fútbol en particular, enseña a los varones a usar dos registros, uno profundamente jerárquico asociado a la casa y al control de ciertas instituciones públicas, como el trabajo, y otro cercano y familiar asociado a la calle. Las mujeres, por el contrario, representan el orden y la jerarquía y no son entrenadas en el lenguaje de la familiaridad interclases. Es por ello que se les acusa de ser más 'machistas' y más 'clasistas' que los varones. Los varones en cambio pueden ser profundamente jerárquicos en la casa y en el trabajo mientras que en la calle, comparten actividades que quiebran estas jerarquías" (1997, p. 118; el subrayado es nuestro).

El texto subrayado permite entender por qué las mujeres perciben más desprecio que los varones. A la situación de menor valor que aún se les sigue atribuyendo en muchos contextos de nuestra sociedad, podemos presumir que se agrega el hecho de que interactúan -entre ellasde acuerdo con un patrón de comportamiento que destaca las diferencias sociales.

Queda aún una última cuestión: la percepción de mayor desprecio entre quienes se consideran más blancos (ver Gráfico 1). El "blanqueamiento" es una de las metas más anheladas por muchos en el Perú (Montoya, 1992): a través de él se espera reconocimiento social y diferenciación con respecto a mestizos, indígenas y negros. Pero, precisamente esa búsqueda de reconocimiento haría que quienes se sienten o son más blancos sean más proclives a interpretar algunas reacciones y conductas del medio circundante como actos discriminatorios hacia ellos. Como lo hemos señalado en otra parte: "quienes están más preocupados por parecer y ser más blancos, o quienes están convencidos de que lo son, son los más proclives a registrar o a calificar conductas hostiles o negativas de los demás hacia ellos como expresiones de discriminación racial. Si 
ellos se ven como más blancos y menos negros que los demás la pregunta que más se formularian sería: ¿por qué se me discrimina? ¿por qué se me trata mal?" (León, 1998; p. 132).

En 1861 Dostoievski dio a la luz Humillados y ofendidos, una de sus grandes novelas. Al igual que casi todos los libros de este novelista, atormentado por una clara conciencia de la dualidad del ser humano $y$ de las tremendas diferencias derivadas de un sistema político injusto Joravsky 1989), Humillados y ofendidos es a la vez una presentación descarnada y una denuncia de la sombría realidad social de la Rusia del siglo pasado; un "subrogado para la politica", como califica Figes (1997; p. 129), a la actividad literaria en el antiguo Imperio de los Zares.

Su obra refleja de modo claro la sociedad en la que él vivió, como también sucede en el caso de Flaubert para la Francia del siglo pasado, o en el de Arguedas para el Perú de esta centuria (Flores Galindo, 1992). Por ello hemos tomado el título de esta novela de Dostoievski para nuestro trabajo.

Humillados y ofendidos: opresores y oprimidos; abusivos y maltratados; señores y sirvientes. Estos son algunas de las realidades antinómicas que pueden ser conjugadas en una sola palabra: injusticia. El desprecio, sentido, visto, vivenciado, practicado o verbalizado: el desprecio en acción o en latencia, es el resultado de esa realidad injusta que es el Perú, un país en el cual se cumple la original afirmación de George Orwell, el asimismo célebre escritor, de que todos somos iguales, pero unos son más iguales que otros (Orwell, 1978).

\section{Referencias}

Adrianzén, A. (1990). Estado y sociedad: señores, masas y ciudadanos. En J. Abugattás, A. Adrianzén, J. de Althaus, F. Jiménez, S. López, y M. Rubio (Eds.), Estado y sociedad: relaciones peligrosas (pp. 13-42). Lima: DESCO. 
Alarcón, R. (1986). Psicologia, pobreza y subdesarrollo. Lima: INIDE

Ames, R. (1995). Entrevista. En F. Silva Santistebán (Ed.), Historia de nuestro tiempo, (pp. 43-54). Lima: Universidad de Lima.

Asociación Laboral para el Desarrollo (1993). Cuestión de piel. Testimonios de racismo en el Perí. Lima.

Asociación Peruana de Estudios e Investigación para la Paz (1990). Violencia estructural en el Perú. Lima.

Barrig, M. y Henriquez, N. [sin fecha]. Presentación. En M. Barrig y N. Henriquez (Comps.), Otras pieles. Género, bistoria y cultura (IX-XVI). Lima: Pontificia Universidad Católica del Perú.

Basadre, J. (1958). La promesa de la vida peruana y otros ensayos. Lima: Librería Editorial Juan Mejía Baca.

Benedict, R. (1987). Raza: ciencia y politica. México, D. F.: Fondo de Cultura Económica.

Ben-Zeev, A. (1987). The nature of emotions. Philosophical Studies, 52, 393409.

Broncano, F. (1995) El control racional de la conducta. En F. Broncano, (Ed.), La mente bumana, (pp. 301-331, vol. 8 Enciclopedia Iberoamericana de la Filosofía). Madrid: Trotta-Consejo Superior de Investigaciones Científicas.

Burga, M. y Flores Galindo, A. (1984). Apogeo y' crisis de la República Aristocrática. Lima: Ediciones Rikchay Perú.

Callirgos, J.C. (1996). Sobre béroes y batallas. Las caminos de la identidad masculina. Lima: Escuela para el Desarrollo.

Callirgos J. C. (1997). El (poco discreto) encanto de la burguesia. Distancias sociales y' discursos legitimadores en el Peri de boy. Lima: Instituto de Defensa Legal. Comisión Fspecial del Senado sobre las Causas de la Violencia y Alternativas de Pacificación en el Perú (1989). I iolencia y pacificación. Iima.

Daridson, J. R. T.; Hughes, D.C.; George, L. K. y Blazer, D. G. (1994). The boundary of social phobia. Exploring the threshold. Archives of General Psychiatry, 51, 975-983.

Dejo Soto, F. (1994). Razas y racismo en Lima. Suplemento Dominical de Fil Comercio, ed. del 13.11. 
Del Aguila Peralta, A. (1997). Callejones y mansiones. Espacios de opinión pública y redes sociales y politicas en la Lima del 900. Lima: Pontificia Lniversidad Católoca del Perú.

Delgado, C. (1974). Problemas sociales en el Perí contemporáneo. Lima: Instituto de Estudios Peruanos.

Duque de la Rochefoucauld. (1984). Reflexiones o sentencias y Máximas morales. Barcelona: Bruguera.

Eguren L.F.; Cano P., J. \& del Aguila A., A. (1997). Eialuación social del desarrollo bumano en el Perú. Lima: Acción Ciudadana.

Evans, M. (1996). ¿Ciudadanos bien informados? Irracionalidad en algunos jóvenes universitarios. Sociedad Anomica, 3 (2), 87-92.

Figes, O. (1997). A people's tragedy. The Russian Revolution 1891-1924. Londres: Pimlico.

Flores Galindo, A. (1988). Tiempo de plagas. Lima: El Caballo Rojo.

Flores Galindo, A. (1992). Dos ensayos sobre José Maria Arguedas. Lima: Sur.

Flores Galindo, A. (1994). Buscando un inca. Identidady utopia en los Andes. Lima: Horizonte.

Fuller Osores, N. J. (1993). Dilemas de la femineidad. Lima: Pontificia Universidad Católica del Perú.

Fuller Osores, N.J. (1997). Identidades masculinas. Varones de clase media en el Perí. Lima: Pontificia Universidad Católica del Perú.

Furnham, A. (1986). Response bias, social desirability and dissimulation. Personality and Individual Differences, 7, 385-400.

Gonzales, O. (1996). Sanchos fracasados. Los arielistas y el pensamiento politico peruano. Lima: PREAL

Harré, R. (1982). El ser social. Una teoría para la psicología social. Madrid: Alianza Editorial.

Heinrichs, H. J. (1997). La ciencia del otro. Humboldt, 121, 54-55.

Hobbes, Th. (1984). Leviatan I, o la materia, forma y poder de una república eclesiástica y civil. Madrid: SARPE.

Izard, C. E. (1991). The psychology of buman emotions. Nueva York: Plenum Press.

Joravsky, D. (1984). Russian psychology: A critical bistory, Oxford: Basil Blackwell. 
Kaplan, H. Y.; Sadock, B. J. y Grebb, J. A. (1996). Sinopsis de psiquiatria. Ciencias de la conducta. Psiquiatria Clinica. Buenos Aires-Baltimore: Panamericana y Williams y Wilkins

Kogan, L. (1996). Ricos y famosos. La clase alta en Lima. Cuestión de Estado. junio-agosto. 18, 11-13.

León, R. (1998). El pais de los extraños. Lima: Universidad Ricardo Palma [en colaboración con J.J. Tan y K. Murillo S.].

León, R.; Delhonte, P.; del Castillo, B. Y León, M. (en preparación). Percepción de la bipocresia en adolescentes: una exploración.

León, R. y Martell, E. (1994). Valoración de conductas verbales y no verbales como expresión de envidia. Revista de Psicologia (PLCP), 12, 191-219.

León, R. y Moscoso, S. (1991). Percepción de la envidia en el Perú en un grupo de estudiantes universitarios de Lima Metropolitana. Retista de Neuro-psiquiatria, 54, 9- 28.

León, R. y Portocarrero, C. (1989). Medición de la autoconciencia en adolescentes de una zona marginal en Lima (Perú). Avances en Psicología Clinica Latinoamericana, 7, 39-54.

León, R. y Sirlopí, D. (1996). Diferencias de género en el Patrón de Conducta Tipo A en un grupo de estudiantes universitarios de I.ima Metropolitaria. Revista de Psicologia, 14, 183-209.

Lersch, Ph. (1968). La estructura de la personalidad. Barcelona: Scientia.

López, S. (1996). Perú: mapas de una ciudadanía inconclusa. Lima: separata especial Contribuciones para pensar el Perí de la revista Sociedad Anómica.

Lópcz, S. (1997). Ciudadanos reales e imaginarios. Concepciones, desarrollo y mapa de la cindadania en el Peri. Lima: Instituto de Diálogo y Propuestas. López Jiménez, M.A. (1994). La organización popular en Lima: de la tradición comunitaria a la participación ciudadana. En T. R. Villasante (Comp.), Las ciudades bablan. Identidades y monimientos sociales en seis metropolis latinoamericanas, (pp. 233-250). Caracas: Nueva Sociedad.

Manrique, N. (1992). Quinientos años después: cambios y permanencias. En N. Manrique, L. M. Glave, F. Muñoz, G. Portocarrero, y E. Trelles (Eds.), 500 años después... eel fin de la bistoria? (pp. 199-214). I.ima: Escuela para el Desarrollo. 199-214. 
Manrique, N. (1993). Notas sobre las condiciones sociales de la violencia política en el Perú. Revista de Neuro-psiquiatria, 56, 235-240.

Matos Mar, J. (1987). Desborde popular y crisis del estado. Lima: Instituto de Estudios Peruanos.

Méndez, C. (1997). República sin indios: la comunidad imaginada del Perú. En H. Urbano (Comp.), Tradición y modernidad en los Andes (pp. 1541). Cuzco: Centro de Estudios Regionales Andinos Bartolomé de las Casas.

Milla Batres, C., (Ed.) (1990). En qué momento se jodió el Peri. Lima: Milla Batres.

Montoya, R. (1992). Al borde del naufragio (Democracia, violencia y problema étnico en el Peris. Lima: Sur.

Nugent, J.C. (1992). El laberinto de la cboledad. Lima: Fundación Friedrich Ebert.

Oliart, P. [sin fecha]. Temidos y despreciados: raza y género en la representación de las clases populares limeñas en la literatura del siglo XIX. En M. Barrig y N. Henríquez (Comps.), Otras pieles. Género, bistoria y cultura (pp. 73-87). Lima: Pontificia Universidad Católica del Perú.

Ortega, J. (1986). Cultura y modernización en la Lima del 900. Lima: Centro de Estudios para el Desarrollo y la Participación.

Orwell, G. (1978). Animal farm. A fairy story. Harmondsworth: Penguin Books.

Plutchik, R. (1987). Las emociones. México, DF: Diana.

Portocarrero, G. (1992). Discriminación social y racismo en el Perú hoy. En N. Manrique, L. M. Glave, F. Muñoz, G. Portocarrero y E. Trelles, (Eds.), 500 años después ... ¿el fin de la historia? (pp. 179-197). Lima: Escuela para el Desarrollo.

Portocarrero, G. (1993). Racismo y mestizaje. Lima: Sur.

Potestá, O. y La Rosa, A. M. (1996). La ciudad desconocida. Fivir Bien, año 1 (5), 12-18.

San Ignacio de Loyola (1982). Obras completas. Madrid: Biblioteca de Autores Cristianos.

Stein, S. y Monge, C. (1988). La crisis del estado patrimonial en el Perí. Lima: Instituto de Estudios Peruanos y Universidad de Miami. 
Tuesta Soldevilla, F. (1997). No sabe/no opina. Encuestas politicas y medios. Lima: Universidad de Lima y Fundación Konrad Adenauer. Twanama, W. (1992). Cholear en Lima. Márgenes, 5 [9], 206-240.

Vargas Llosa, M. (1996). La utopía arcaica. José Maria Arguedas y las ficciones del indigenismo. México,D. F.: Fondo de Cultura Económica.

Warren, H. C., (1963). Diccionario de psicologia. México, D. F.: Fondo de Cultura Económica.

Wills, Th. A. (1991). Social comparison processes in coping and health. En C. R. Snyder y D. R. Forsyth (Eds.), Handbook of Social and Clinical Psychology (pp. 376-394). Nueva York: Pergamon Press. 
Humillados $y$ ofendidos

Anexo: La Escala de percepción de desprecio en el periu, marco referencial, elaboración y validación

En el presente trabajo, cuando hablamos de desprecio nos referimos a una variedad de afectos que incluye al desprecio sensu stricto, el menosprecio, el desdén y la discriminación.

Por desprecio sensu stricto entendemos la negación del valor intrínseco de una persona, que puede llegar inclusive a ignorarla por completo en su realidad física y tanto más en su significado social. El menosprecio es la valoración del otro como alguien de escaso de valor o de reducidas capacidades; frente a él nos sentimos decididamente superiores. Tanto en el desprecio sensu stricto como en el menosprecio hay un proceso de comparación social hacia abajo (downward comparison) que nos coloca en situación de superioridad.

El desdén, más que una actitud, es el resultado del menosprecio, y supone indiferencia hacia el otro, mínimo interés hacia él y, por tanto, muy reducida posibilidad de interacción. Rom Harré, cn su excelente El ser social (1982), señala que "el desprecio [...] se demuestra por el desdén y se corresponde con el envilecimiento" (p. 41).

Por último, la discriminación es la activa postergación, la premeditada marginación de determinadas personas con respecto al acceso o ejercicio de derechos u oportunidades. En el Perú, Twanama (1992) se ha referido a la discriminación como el "desigual trato que se da a individuos o grupos basados en características tales como el sexo, la etnicidad, los antecedentes culturales" (p. 212).

No es muy amplia la bibliografía existente sobre estos afectos. Lersch (1968) se ha referido al desprecio desde una perspectiva fenomenológica. El análisis más detenido es, sin embargo, el llevado a cabo por Izard, en su The psychology of buman emotions (1991). Izard se refiere al menosprecio 
(contempt), y considera a éste, junto con el disgusto y el enojo como la triada de la hostilidad, origen para muchas formas de conducta agresiva. Plutchik, en Las emociones (1987), hace también algunas menciones al desprecio.

El desprecio y la discriminación constituyen asimismo temas abordados en estudios acerca del racismo, que es, como lo señala Ruth Benedict (1987), "un modo pretencioso de decir yo soy de los mejores" ( $\mathrm{p}$. 125).

Como todo afecto, el desprecio y las variantes de él mencionadas tienen tres niveles de descripción: el neurofisiológico-bioquímico; el motor o comportamental; y el mental (Ben-Zeev 1987). El primer nivel es quizás el que más enigmas encierra: ¿se producen fenómenos fisiológicos tales como mayor descarga de adrenalina, cambios en la actividad gastrointestinal o en la circulación sanguínea, asociados con el desprecio o con la sensación de ser despreciado? No lo sabemos, pero se sospecha que algunos aspectos de las emóciones están vinculados con ciertas hormonas $\mathrm{y}$ algunos neurotransmisores (como las dopaminas y endorfinas) (Broncano 1995). Es plausible suponer que algo así, de modo sutil, ocurre también con el desprecio, aunque Izard (1991) señala que en él hay la menor cantidad de activación fisiológica: "mientras que el enojo es una emoción de relativa alta excitación, el desprecio es más bien una emoción fría. Esto significa que hay probablemente muy poco cambio en la tasa cardiaca y en la respiración así como en otras funciones corporales que se modifican más bien de modo dramático en niveles moderados e intensos de enojo" (p. 272).

La expresividad de los afectos es, a no dudarlo, lo que más nos impacta y lo que delata su presencia. Ya en el siglo XVII, el Duque de la Rochefoucauld señalaba que "cada sentimiento tiene un tono de voz, unos ademanes y un semblante que le es propio" (1984; p. 65). Es evidentemente, en el plano comportamental en el cual se reconoce con más clarida del desprecio: la mirada dirigida a la lejanía y no al interlocutor; la inexpresividad; la nula disposición a establecer contacto social con él; 
las respuestas monosilábicas o el silencio absoluto, son algunas de las más manifiestas.

Sin embargo, es necesario anotar que algunas de esas conductas ocurren también en personas que padecen de fobia social (Kaplan et al. 1996). Pero, mientras que los fóbicos sociales presentan además severos problemas para conseguir y mantener el trabajo y para tener éxito en la educación (Davidson et al. 1994), no ocurre lo mismo con aquellos que desprecian a alguien. Kaplan et al. (1996) se refieren a las personas dominantes que "tienden a caminar con sus mentones alzados" (p. 610). Es Lersch quien ofrece la descripción más precisa al señalar que tanto en la estima como en el desprecio "nos quedamos siempre donde estábamos, a una adecuada distancia del objeto de la emoción" (1968; p. 221). "Además constituye la configuración mocional del desprecio el gesto virtual del arrojar-lejos-de-sí o del borrar-del-campo-visual. En el desprecio miramos al otro por encima o miramos más allá de ćl porque no lo consideramos digno de acompañarnos en nuestra convivencia", concluye.

El plano mental es de gran complejidad. En él intervienen una serie de factores, demasiados para detenernos en ellos, razón por la cual habremos sólo de enumerarlos: escala de valores referida a rasgos aceptables e inaceptables en las otras personas; antipatía; rechazo, procesos de comparación social, enojo. Izard (1991) se refiere en detalle a ellos. De acuerdo con Plutchik (1987) el desprecio proviene de una interrelación de enojo y repugnancia; un elemento constitutivo es, asimismo, la indiferencia. Sobre el particular vale la pena recordar la definición de desprecio que ofrece Thomas Hobbes (1588-1679), en su célebre Leiratán (1984): "Aquellas cosas que no deseamos ni odiamos decimos que son despreciadas: el desprecio no es otra cosa que una inmovilidad o contumacia del corazón, que resiste a la acción de ciertas cosas; se debe a que el corazón resulta estimulado de otro modo por objetos cuya acción es más intensa, o por falta de experiencia respecto a lo que despreciamos" (p. 69). 
En el reactivo que hemos elaborado nos concentramos sobre todo en el plano de lo comportamental.

\section{Elaboracion del reaction}

Se elaboró una lista de once afirmaciones referidas a la vivencia de haber sido discriminado (A); a la observación de que otros eran discriminados (C,D,F); a la atribución a personas de otros departamentos de los problemas de Lima (B); a la valoración de la sociedad peruana como despreciativa $(G, H)$; a la idea de que es bueno mantener distancias con los demás (E); a las ventajas comparativas de ser blanco en la sociedad peruana $(\mathrm{J}, \mathrm{K})$; y a la valoración negativa de algunas prácticas discriminatorias en el Perú (I).

Las once afirmaciones buscaban evaluar un aspecto amplio del desprecio, la discriminación y la superioridad social / racial sentida, observada, vivenciada y conceptualizada. Para ellas sc elaboró un sistema de respuestas de cinco posibilidades: Totalmente falso; Bastante falso; No sé; Bastante cierto; y, Totalmente cierto.

La lista fue aplicada a 789 universitarios ( 362 hombres y 427 mujeres) de Lima Metropolitana, de modo anónimo y colectivo. Tras un proceso de depuración basado en varios criterios se consideraron 617 sujetos. Se procedió seguidamente a obtener estadísticas básicas de los resultados, que incluian las correlaciones ítem-total, a fin de proceder a la validación estadística. Los resultados se presentan en el Cuadro 5:

Los items A, B, E, F, I arrojan correlaciones item-total muy por debajo de $\mathrm{r} .=.30$, que se suele considerar como apropiada para procesos de validación como éste. El alfa (.56) resulta asimismo poco satisfactorio. Por ese motivo, decidimos descartar los cinco ítems antes enumerados, para volver a calcular la correlación ítem-total de los restantes. Los resultados se dan a conocer en el Cuadro 5. 
Humillados y ofendidos

\section{Cuadro 5}

Correlación item - total y determinación del alfa de Cmonbach de los once items de la lista inicial.

\begin{tabular}{|ccc|}
\hline $\begin{array}{c}\text { Item } \\
\text { A }\end{array}$ & $\begin{array}{c}\text { Correlación } \\
\text { item total }\end{array}$ & $\begin{array}{c}\text { alfa } \\
\text { si se omite el item }\end{array}$ \\
\hline B & .18 & .56 \\
C & .10 & .58 \\
D & .33 & .51 \\
E & .28 & .53 \\
F & .13 & .57 \\
G & .22 & .55 \\
H & .43 & .50 \\
I & .41 & .24 \\
J & .03 & .59 \\
K & .35 & .52 \\
$n=617$ & .34 & .51 \\
\hline
\end{tabular}

En este nuevo tratamiento estadístico las correlaciones de los seis ítems se encuentran por encima de $r .=.30$, y el alpha de Cronbach se ha elevado a .6344 , aceptable para el empleo del reactivo en trabajos de investigación.

\section{Cuadro 6}

Correlación item - total. determinacion del alfa de Cronbach de scis items (sumunda rersión)

\begin{tabular}{|ccc|}
\hline Item & Correlación & $\begin{array}{c}\text { alfa } \\
\text { item-total }\end{array}$ \\
\hline $01(\mathrm{C})$ & .33 & si se omite el item \\
$02(\mathrm{D})$ & .35 & .62 \\
$03(\mathrm{G})$ & .41 & .60 \\
$04(\mathrm{H})$ & .46 & .58 \\
$05(\mathrm{~J})$ & .38 & .59 \\
$06(\mathrm{~K})$ & .34 & .61 \\
\hline$n=617$ & alfa: .634 & \\
\hline
\end{tabular}


El promedio de puntajes para toda la muestra fue de 22.94 (DE= 3.88). En el Cuadro 7 se presentan normas provisionales expresadas en percentiles.

\section{Cuadro 7}

Correlacion item - total y determinación del alfa de Cronbacb de seis items (segunda versión)

\begin{tabular}{|cccc|}
\hline Percentil & Valor & Frecuencia & $\%$ \\
\hline & 7 & 2 & .3 \\
& 8 & 1 & .1 \\
& 9 & 1 & .1 \\
& 10 & 2 & .3 \\
& 11 & 3 & .4 \\
& 12 & 3 & .4 \\
& 13 & 2 & .3 \\
& 14 & 4 & .5 \\
& 15 & 6 & .8 \\
& 16 & 17 & 2.2 \\
10 & 17 & 31 & 3.9 \\
& 18 & 21 & 2.7 \\
20 & 19 & 40 & 5.1 \\
30 & 20 & 53 & 6.7 \\
40 & 21 & 56 & 7.1 \\
50 & 22 & 73 & 9.3 \\
60 & 23 & 88 & 11.2 \\
70 & 24 & 82 & 10.4 \\
80 & 25 & 76 & 9.6 \\
& 26 & 67 & 8.5 \\
90 & 27 & 57 & 7.2 \\
& 28 & 37 & 4.7 \\
& 29 & 21 & 2.7 \\
& 30 & 22 & 2.8 \\
\hline & NR & 24 & 3.0 \\
\hline
\end{tabular}


Humillados y ofendidos

\section{Ciuadro 8}

Escala de Percepción de Desprecio en el Perí

\begin{tabular}{|c|c|c|}
\hline $\begin{array}{l}\text { Versión inicial } \\
\text { Item }\end{array}$ & $\begin{array}{c}\text { Versión final } \\
\text { Item }\end{array}$ & Afirmación \\
\hline A & - & $\begin{array}{l}\text { He tenido la impresión (al menos en } \\
\text { una oportunidad) de haber sido discri- } \\
\text { minado/a por el color de mi piel. }\end{array}$ \\
\hline B & - & $\begin{array}{l}\text { Los problemas de Lima se deben en } \\
\text { gran parte al hecho de que hay acá mucha } \\
\text { gente de provincias. }\end{array}$ \\
\hline $\mathrm{C}$ & 01 & $\begin{array}{l}\text { Entre mis amigos hay personas que } \\
\text { desprecian o tratan mal a personas } \\
\text { negras o de los Andes. }\end{array}$ \\
\hline $\mathrm{D}$ & 02 & $\begin{array}{l}\text { He observado más de una vez que } \\
\text { alguien era discriminado por su raza o } \\
\text { por cl color de su piel. }\end{array}$ \\
\hline $\mathrm{E}$ & - & $\begin{array}{l}\text { Creo que es bueno "darse su lado" } \\
\text { con los demás. }\end{array}$ \\
\hline $\mathrm{F}$ & - & $\begin{array}{l}\text { Me he dado cuenta de que algunas } \\
\text { veces trataba a alguien con un poco de } \\
\text { desprecio debido al color de su piel. }\end{array}$ \\
\hline G & 03 & Acá la gente es muy despreciativa. \\
\hline $\mathrm{H}$ & 04 & $\begin{array}{l}\text { En el Perú la gente blanca desprecia y } \\
\text { "basurea" a la de raza negra o de los } \\
\text { Andes. }\end{array}$ \\
\hline I & - & $\begin{array}{l}\text { Me parece que está mal que alguna } \\
\text { gente quiera alquilar su casa sólo a } \\
\text { extranjeros (norteamericanos, ingleses). }\end{array}$ \\
\hline J & 05 & $\begin{array}{l}\text { Siento que en determinados contextos } \\
\text { las personas blancas tienen acceso a } \\
\text { mejores oportunidades de vida. }\end{array}$ \\
\hline $\mathrm{K}$ & 06 & $\begin{array}{l}\text { Cuando en un aviso sc solicita "buena } \\
\text { presencia" me parece que cl color de la } \\
\text { piel de quicn se presente juega un rol } \\
\text { importante. }\end{array}$ \\
\hline
\end{tabular}

